\title{
Skyrmions up to Baryon Number 108
}

\author{
D.T.J. Feist*, P.H.C. Lau ${ }^{\dagger}$ and N.S. Manton \\ Department of Applied Mathematics and Theoretical Physics \\ University of Cambridge \\ Wilberforce Road, Cambridge CB3 0WA, England
}

October 2012

\begin{abstract}
The Skyrme crystal is built up of repeating units similar to the cubic Skyrmion of baryon number 4. Using this as guide, we construct new Skyrmion solutions in the massive pion case, with various baryon numbers up to 108. Most of our solutions resemble chunks of the Skyrme crystal. They are constructed using a multi-layer version of the rational map ansatz to create initial configurations, which are then relaxed numerically to find the energy minima. The coefficients of the rational maps are found by a geometrical construction related to the Skyrme crystal structure. We find some further solutions by numerical relaxation of clusters composed of baryon number 4 Skyrmions.
\end{abstract}

\footnotetext{
*D.Feist@damtp.cam.ac.uk

†P.H.C.Lau@damtp.cam.ac.uk

¥N.S.Manton@damtp.cam.ac.uk
} 


\section{Introduction}

The Skyrme model was proposed in 1961 by THR Skyrme as a model for nuclear physics [1, 2, 3. It is a nonlinear field theory of pions admitting solitonic solutions called Skyrmions. Skyrmions model atomic nuclei, and have a conserved, integer-valued topological charge $B$, which is interpreted as the baryon number, or mass number, of a nucleus.

The Skyrme field $U(x)$ is an $S U(2)$-valued scalar field. It can be written as

$$
U(x)=\sigma(x) \boldsymbol{I}+i \boldsymbol{\pi}(x) \cdot \boldsymbol{\tau}
$$

with pion fields $\boldsymbol{\pi}=\left(\pi_{1}, \pi_{2}, \pi_{3}\right)$ and sigma field $\sigma$. Here $x=(t, \mathbf{x})=\left(x_{0}, x_{1}, x_{2}, x_{3}\right)$ and $\boldsymbol{\tau}$ are the Pauli matrices. $\sigma$ and $\boldsymbol{\pi}$ are not independent as $U U^{\dagger}=\left(\sigma^{2}+\boldsymbol{\pi} \cdot \boldsymbol{\pi}\right) \boldsymbol{I}=\boldsymbol{I}$.

The Lagrangian, in "Skyrme units", is

$$
L=\frac{1}{12 \pi^{2}} \int\left\{-\frac{1}{2} \operatorname{Tr}\left(R_{\mu} R^{\mu}\right)+\frac{1}{16} \operatorname{Tr}\left(\left[R_{\mu}, R_{\nu}\right]\left[R^{\mu}, R^{\nu}\right]\right)-m^{2} \operatorname{Tr}(\boldsymbol{I}-U)\right\} \mathrm{d}^{3} x,
$$

where $R_{\mu}=\left(\partial_{\mu} U\right) U^{\dagger}$ is the right current. The energy unit is roughly $700 \mathrm{MeV}$ and the length unit is roughly $1 \mathrm{fm}$. The parameter $m$ is the pion mass in Skyrme units. Using the physical pion mass one finds $m \simeq 0.5$ [4], but this is sensitive to the length unit. It has been found that if one calibrates the model to real spinning nucleons, and some larger nuclei like Carbon-12 in its ground and excited states, a value of $m=1$ gives a better fit [5, 6, 7]. Therefore $m=1$ is used in this paper.

For a static field $U(\mathbf{x})$, the energy depends only on $U$ and its spatial derivatives encoded in the spatial current $R_{i}$, and is

$$
E=\frac{1}{12 \pi^{2}} \int\left\{-\frac{1}{2} \operatorname{Tr}\left(R_{i} R_{i}\right)-\frac{1}{16} \operatorname{Tr}\left(\left[R_{i}, R_{j}\right]\left[R_{i}, R_{j}\right]\right)+m^{2} \operatorname{Tr}(\boldsymbol{I}-U)\right\} \mathrm{d}^{3} x .
$$

$U=\boldsymbol{I}$ is the vacuum of the theory. For a finite energy field configuration, it is necessary that $U \rightarrow \boldsymbol{I}$ as $|\mathbf{x}| \rightarrow \infty$, hence $\sigma \rightarrow 1$ and $\boldsymbol{\pi} \rightarrow \mathbf{0}$.

The baryon number $B$ is the topological degree of the map $U: \mathbb{R}^{3} \rightarrow S U(2)$, which is well-defined because $U \rightarrow \boldsymbol{I}$ at spatial infinity. $B$ can be defined as the integral over $\mathbb{R}^{3}$ of the baryon density

$$
\mathcal{B}=-\frac{1}{24 \pi^{2}} \epsilon_{i j k} \operatorname{Tr}\left(R_{i} R_{j} R_{k}\right)
$$

The minimum energy field configurations for each $B$ are the true Skyrmions. For all but the smallest baryon numbers one finds further configurations that are local minima of the energy, with very similar energies, and these are also called Skyrmions.

The Skyrme model with massless pions $(m=0)$ has been studied intensively, and Skyrmions with all baryon numbers up to 22 have been found [8]. Beyond baryon numbers 1 and 2, these Skyrmions have a polyhedral shell-like structure, surrounding a hollow 
region of small baryon density whose volume increases proportionally to the baryon number. This does not model real nuclei well. In recent years, the Skyrme model has been studied with a pion mass $m$ around 1 , and Skyrmions with selected baryon numbers up to $B=32$ have been found [5, 9, 10]. These massive pion solutions are closer to the structure of real nuclei. They are more compact, and clustering can be observed. For example, the $B=8$ Skyrmion consists of two $B=4$ Skyrmions, as in the $\alpha$-particle model of nuclei. The reason for this greater compactness is that in the hollow region of the shell-like Skyrmions, $U$ is close to $-\boldsymbol{I}$, and the pion mass term makes this unstable.

Many Skyrmions have been found with the help of the rational map ansatz [11]. A rational map is a quotient of two complex polynomials $p(z) / q(z)$, and using stereographic projection it can be interpreted as a map from the Riemann sphere, $S^{2}$, to itself. A rational map is an exact multi-lump solution of the $O(3)$ sigma model on $S^{2}$, this being a Skyrme-type model in two dimensions. In three dimensions, the rational map is used to encode the angular part of a Skyrmion, and by extending it using a radial profile function, one gets useful field configurations of the three-dimensional Skyrme model. Part of the Skyrme energy depends on the coefficients of the rational map and it is important to optimise the coefficients by minimising the energy, or at least by getting close to the minimum. After this, a full numerical relaxation quickly leads to a shell-like Skyrmion, with the baryon number equal to the degree of the map. The approach can be extended to a multi-layer ansatz using two or more rational maps [12], and then, with $m=1$, numerical relaxation leads to the more compact Skyrmions.

Rational maps can be constructed with the conjectured symmetry of a Skyrmion for a given baryon number. This is helpful, although one should check that configurations with different symmetries are not of lower energy. The coefficients of a rational map are constrained by its symmetry, and for small degrees only certain symmetries are allowed. For example, for degree 4 there is an essentially unique rational map with cubic symmetry, and no map with icosahedral symmetry. This goes some way to explaining the cubic symmetry of the $B=4$ Skyrmion. However, for larger baryon numbers, symmetry does not fix the coefficients uniquely. The remaining undetermined coefficients can be found by numerical optimisation of the relevant part of the Skyrme energy [8], but this proves to be very time consuming and ineffective for baryon numbers beyond about 20. New techniques to construct near-optimal rational maps are presented in this paper, and several new Skyrmion solutions have been found using these. They have various baryon numbers up to $B=108$, far higher than those of Skyrmions found before. The closest comparable solution, previously known, is the cubic $B=32$ Skyrmion, which can be obtained using a double rational map ansatz [10].

A feature of many Skyrmions with massive pions is that they look like fields cut out from the infinite Skyrme crystal. We call these crystal chunks. The crystal with massless pions has a primitive cubic structure where each unit cell contains half a unit of baryon number, and can be regarded as containing a half-Skyrmion [13, 14, 15]. In neighbouring unit cells the fields repeat with an $S U(2)$ twist. There is exact periodicity after two lattice 
spacings, so a true cubic unit cell contains eight half-Skyrmions, and hence four units of baryon number. In the crystal with massive pions, the half-Skyrmion symmetry is slightly broken. The true unit cell remains a cube with four units of baryon number. The fields in this unit cell are very similar to the isolated $B=4$ Skyrmion. As a consequence, many Skyrmion solutions with baryon number a multiple of four are crystal chunks. At the same time they look like clusters of $B=4$ Skyrmions glued together, analogous to what is expected in the $\alpha$-particle model [10].

The geometrical method used to construct rational maps in this paper is based on the Skyrme crystal. The zeros and poles of the rational map are derived from the locations of half-Skyrmions in the crystal [16]. This requires a conversion of the Cartesian coordinates in the crystal lattice (relative to a suitable origin) to angular coordinates, and then to the Riemann sphere coordinate $z$. The simplest rational maps acquire the cubic point symmetry of the crystal, but these have restricted values for their degrees, resulting in Skyrmions with a restricted set of baryon numbers. However, by selecting subsets of the half-Skyrmions, and by applying a corner-cutting technique that we will explain below, we are able to construct a large range of useful rational maps with lower symmetry. This yields a larger set of baryon numbers. We would like to construct Skyrmions with every possible baryon number up to $B=300$, as this would potentially deal with all nuclei. However, this remains beyond our reach.

While some of the solutions we find are composed of $B=4$ Skyrmions, there are some exceptions. Two new solutions with $B=20$ have $T_{d}$ and $D_{2 h}$ symmetry, respectively. Both solutions consist of four $B=4$ Skyrmions with four $B=1$ Skyrmions between them, rather than of five $B=4$ Skyrmions. This resembles the $4 \alpha+4 n$ cluster structure recently suggested for ${ }^{20} \mathrm{O}[17$.

A few solutions presented here were not constructed using a rational map ansatz. Instead they were found by relaxing an initial configuration made from a number of $B=4$ Skyrmions glued together using the product ansatz [2]. The $B=24$ solutions we have obtained this way are of lower energy than anything constructed using the rational map ansatz.

In Section 2 we recall the basic $B=1$ hedgehog Skyrmion, and the colouring scheme for pion fields that has been found useful before, and is used again here. The rational map ansatz and the multi-layer rational map ansatz are also recalled. In Section 3 we describe the geometrical method, based on the Skyrme crystal structure, for constructing many useful rational maps. New Skyrmion solutions obtained by this method are also discussed, including the Skyrmions of highest baryon number that we have found, with $B \geq 100$. Section 4 analyses a class of rational maps with cubic or tetrahedral symmetry, and some related Skyrmions. Section 5 describes the two $B=24$ Skyrmions that we have obtained as clusters of six $B=4$ Skyrmions. Concluding remarks are in Section 6, and our numerical methods are discussed in the Appendix. 


\section{$2 \quad$ Hedgehogs and rational maps}

\section{1 $B=1$ Skyrmion and colouring scheme}

The $B=1$ Skyrmion has an $O(3)$ symmetry (combined rotations in real space and among the pion fields, together with an inversion symmetry). It looks like a hedgehog in that the pion fields are pointing radially outward from the centre. The ansatz for this field configuration is

$$
U(\mathbf{x})=\exp (i f(r) \hat{\mathbf{x}} \cdot \boldsymbol{\tau}),
$$

where $r=|\mathbf{x}|$ and $\hat{\mathbf{x}}=\mathbf{x} / r$. This results in $\sigma=\cos f(r), \boldsymbol{\pi}=\sin f(r) \hat{\mathbf{x}}$, and to get a $B=1$ configuration, the boundary conditions

$$
f(0)=\pi, \quad f(\infty)=0
$$

have to be imposed. Optimising the radial profile function $f(r)$, to minimise the energy, gives the Skyrmion. Table 1 lists the energies and other properties of this and other Skyrmions that we have found.

To visualise Skyrmions, a surface of constant baryon density is plotted, coloured using P.O. Runge's colour sphere. The colours indicate the value of the normalised pion field $\hat{\boldsymbol{\pi}}=\boldsymbol{\pi} /|\boldsymbol{\pi}|$. No attempt at colouring is made at points where $\sigma= \pm 1$ and $\boldsymbol{\pi}=0$, but these are absent from the surfaces we show. The equator of the colour sphere corresponds to $\hat{\boldsymbol{\pi}}_{3}=0$. Here, the primary colours red, green and blue show where the field $\hat{\boldsymbol{\pi}}_{1}+i \hat{\boldsymbol{\pi}}_{2}$ takes the values $1, \exp (i 2 \pi / 3)$ and $\exp (i 4 \pi / 3)$, and the intermediate colours yellow, cyan and magenta show the values $\exp (i \pi / 3),-1$ and $\exp (i 5 \pi / 3)$. The $\hat{\boldsymbol{\pi}}_{3}$-value is assigned to the "lightness" colour attribute so that white and black, at the poles on the colour sphere, show where $\hat{\boldsymbol{\pi}}_{3}= \pm 1$, respectively.

The hedgehog form of the $B=1$ Skyrmion means that on a spherical surface, the colouring reproduces the colour sphere itself (see Figure 1).

Because the pion fields are scalar fields, charges (sources) of equal sign attract. As a consequence, parts of Skyrmions with the same colour tend to attract, and low energy configurations can be constructed by gluing Skyrmions together with colours matching. This is what makes the colouring so useful. However for $B>1$ there is some frustration, that is, non-matching colours, as one can see from some of the figures in this paper. If there were no frustration, as occurs for example with a $B=1$ and $B=-1$ Skyrmion pair, then the Skyrmions could annihilate. 


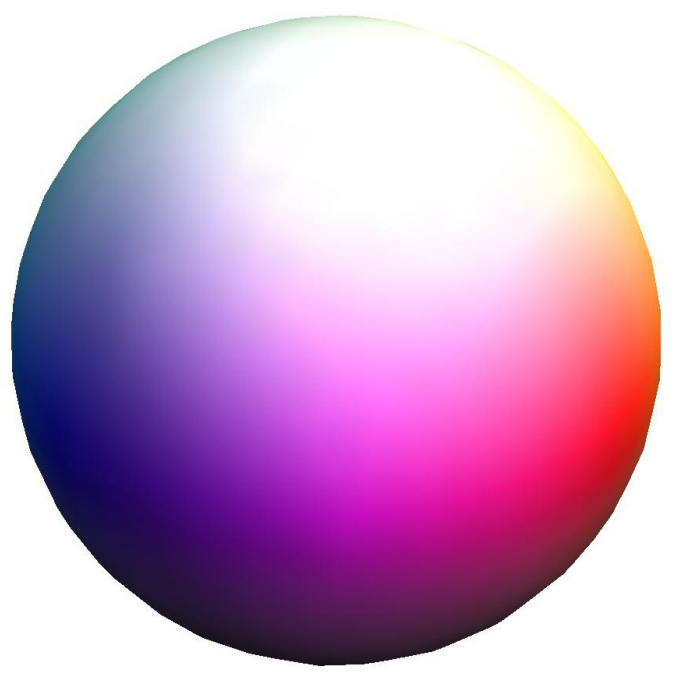

Figure 1: Colour sphere, and $B=1$ Skyrmion

\subsection{Single rational map ansatz}

The rational map ansatz [11] generates approximate Skyrmions with separated radial and angular dependence, generalising the hedgehog ansatz (2.1). The radial part is again given by a profile function $f(r)$ satisfying the boundary conditions $f(0)=\pi$ and $f(\infty)=0$. It is assumed that $f(r)$ decreases monotonically as $r$ increases. The angular part is determined by a rational function $R(z)=p(z) / q(z)$, where $p(z)$ and $q(z)$ are polynomials. Here $z$ is the complex coordinate on the Riemann sphere defined, via stereographic projection, as $z=\tan \left(\frac{1}{2} \theta\right) \exp (i \phi)$, where $\theta$ and $\phi$ are the usual spherical polar coordinates. The $z$-coordinate is related to the Cartesian coordinates on the unit sphere $S^{2} \subset \mathbb{R}^{3}$ via the formula

$$
z=\frac{\hat{\mathbf{n}}_{1}+i \hat{\mathbf{n}}_{2}}{1+\hat{\mathbf{n}}_{3}},
$$

where $\hat{\mathbf{n}}$ is the outward normal vector on the unit sphere. The inverse of this formula is

$$
\hat{\mathbf{n}}_{z}=\frac{1}{1+|z|^{2}}\left(z+\bar{z}, i(\bar{z}-z), 1-|z|^{2}\right) .
$$

The rational map ansatz, combining the rational map $R(z)$ with the radial profile function $f(r)$, is defined by

$$
\begin{aligned}
U(r, z) & =\exp \left(i f(r) \hat{\mathbf{n}}_{R(z)} \cdot \boldsymbol{\tau}\right) \\
& =\cos f(r) \boldsymbol{I}+i \sin f(r) \hat{\mathbf{n}}_{R(z)} \cdot \boldsymbol{\tau},
\end{aligned}
$$

where $\hat{\mathbf{n}}_{R(z)}$ is defined analogously to (2.4). This generalises the hedgehog ansatz (2.1), which can be recovered by setting $R(z)=z$.

The baryon number $B$ is the topological degree of the rational map $R: S^{2} \rightarrow S^{2}$, which is also the algebraic degree of $R(z)$, the higher of the degrees of the polynomials 
$p(z)$ and $q(z)$. Below are some of the well known rational maps of high symmetry which approximate the Skyrmions with baryon numbers $B=1,2,3,4$ :

$$
\begin{aligned}
R(z) & =z, & R(z) & =z^{2}, \\
R(z) & =\frac{z^{3}-\sqrt{3} i z}{\sqrt{3} i z^{2}-1}, & R(z) & =\frac{z^{4}+2 \sqrt{3} i z^{2}+1}{z^{4}-2 \sqrt{3} i z^{2}+1} .
\end{aligned}
$$

Optimising the profile function $f(r)$ gives approximate Skyrmions, but true Skyrmions can only be found by further numerical relaxation. The true Skyrmions have the same symmetries as their rational maps, but for $B>1$ their angular dependence does vary with radius, contrary to what the rational map ansatz allows. The symmetry groups are $O(3), D_{\infty h}, T_{d}, O_{h}$ for $B=1,2,3,4$ respectively. The $B=3$ and $B=4$ Skyrmions are shown in Figure 2 .
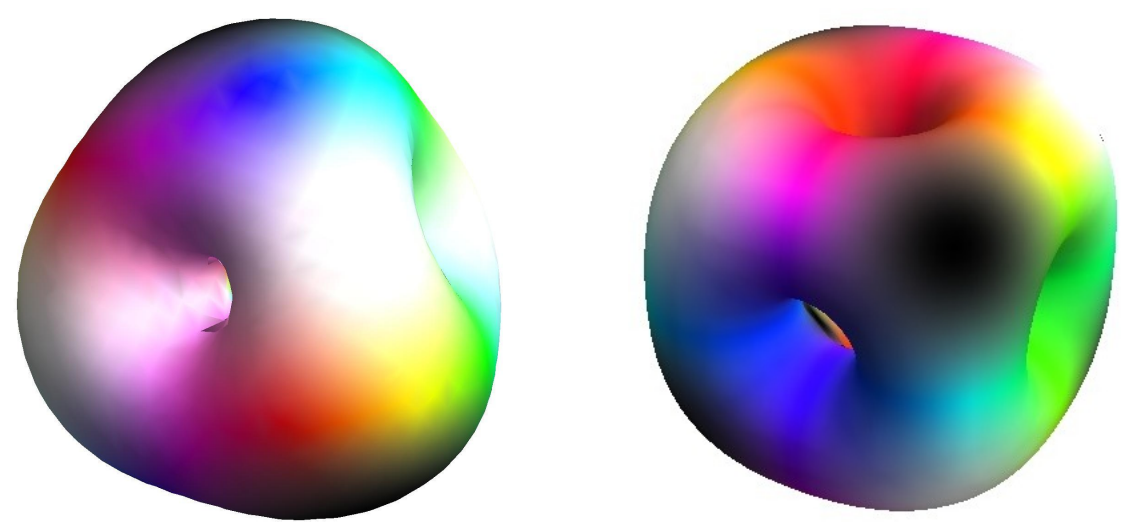

Figure 2: $B=3$ tetrahedral (left) and $B=4$ cubic (right) Skyrmions

\subsection{Multi-layer rational map ansatz}

The single rational map ansatz works well for Skyrmions of small baryon number, but for larger Skyrmions, a multi-layered structure is needed. The double rational map ansatz was the first extension to be considered [12]. It uses two rational maps denoted $R^{\text {in }}(z)$ and $R^{\text {out }}(z)$. A monotonic profile function $f(r)$ is needed, taking an extended range of values with $f(0)=2 \pi$ and $f(\infty)=0$. Let $r_{1}$ be the radius where $f\left(r_{1}\right)=\pi$. The ansatz is the same as $(2.5)$, but $R(z)=R^{\text {in }}(z)$ for $0 \leq r \leq r_{1}$ and $R(z)=R^{\text {out }}(z)$ for $r_{1} \leq r<\infty$. On the sphere of radius $r_{1}$, the Skyrme field is $U=-\boldsymbol{I}$. The total baryon number is the sum of the degrees of the maps $R^{\text {in }}$ and $R^{\text {out }}$.

In the $K$-layer ansatz, the profile function needs to take the values $f(0)=K \pi, f(\infty)=$ 0 with the $k$-th rational map $R^{k}(z)$ used in the region $r_{k-1} \leq r \leq r_{k}$ for $k=1,2, \cdots, K$. Here $f\left(r_{k}\right)=(K-k) \pi$, with $r_{0}=0, r_{K}=\infty$. The total baryon number is the sum of the degrees of all the rational maps. 
It is best if the maps in the different layers share a substantial amount of symmetry. The maps in neighbouring layers need to be carefully oriented so that colours match as far as possible. This requirement fixes certain relative signs that are not determined by symmetry alone. The multi-layer ansatz is useful, but not so close to Skyrmion solutions as the single layer version. After relaxation, the solutions do not have $U= \pm \boldsymbol{I}$ on complete spheres, but instead at isolated points.

\section{Geometrical construction of rational maps}

\subsection{Skyrmions from the Skyrme crystal}

Rational maps of low degree can be constructed using symmetry algebra to fix the coefficients of the polynomials $p(z)$ and $q(z)$. Generally, however, symmetry leaves some coefficients undetermined, and these have in the past been found numerically, by minimising an expression that arises when the rational map ansatz is inserted into the Skyrme energy [11]. For degrees beyond about 20, however, this minimisation become intractible, and a new approach is needed.

The Skyrme crystal, for massless pions, is made of half-Skyrmions [13, 14, 15]. The field values at lattice points of the crystal are known precisely, because of the crystal symmetry constraints. In a convenient orientation, the $\hat{\boldsymbol{\pi}}_{3}$ field takes the values \pm 1 at alternating lattice points (white and black in our colour scheme). These values correspond to the zeros and poles of a rational map $R(z)$, that is, to the roots of the polynomials $p(z)$ and $q(z)$.

This observation [16] is the basis for a geometrical construction of a range of useful rational maps, which lead to new Skyrmions. In particular, the construction leads to the cubic Skyrmions with baryon number $B=4 n^{3}$, with $n \in \mathbb{N}^{+}$, resembling chunks of the Skyrme crystal. The first construction of the $B=32$ Skyrmion (the $n=2$ example) was by manipulating the field of the Skyrme crystal directly [18]. However, it is easier to use the intermediary of an $n$-layer rational map ansatz. The real advantage of the rational map approach is that one can fairly easily vary the degrees of the maps and generate Skyrmions with baryon numbers not of the form $4 n^{3}$.

Here is how we rederived the $B=32$ Skyrmion. Figure 3 shows the $4 \times 4 \times 4$ grid of half-Skyrmion locations that occur in a cubic chunk of the crystal. The (black) circles and (blue) squares are used as the zeros and poles, respectively, for the construction of the rational maps. The grid needs to be separated into two layers. The outer layer has 56 points and the inner (hidden) layer has $2 \times 2 \times 2=8$ points. The corresponding rational maps have degrees 28 and 4 .

We set up scaled Cartesian coordinates $\left(y_{1}, y_{2}, y_{3}\right)$, with the origin at the centre of the 

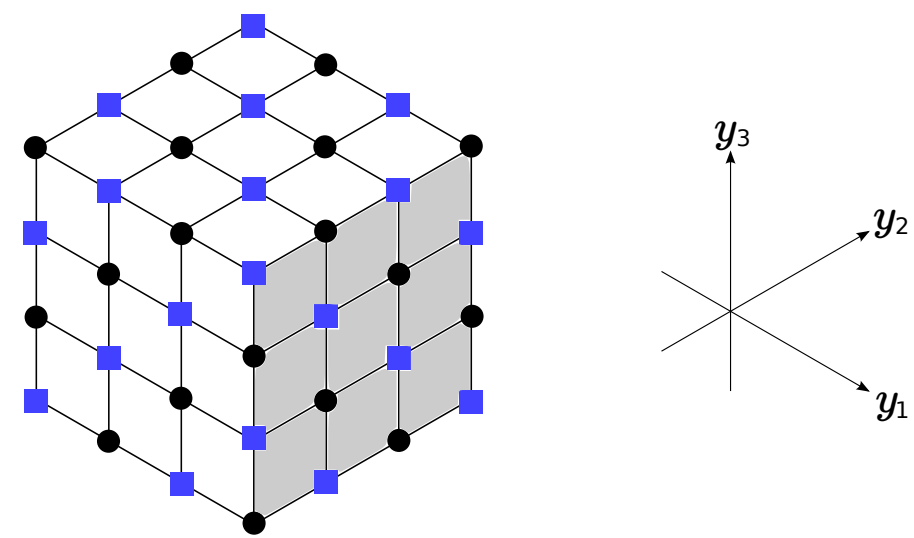

Figure 3: $4 \times 4 \times 4$ Cubic grid

chunk, and lattice points having half-integer coordinates. The distance to the origin is denoted by $\rho$. For example, the inner layer has its eight points at $( \pm 1 / 2, \pm 1 / 2, \pm 1 / 2)$, with $\rho=\sqrt{3} / 2$. The outer corner points are at $( \pm 3 / 2, \pm 3 / 2, \pm 3 / 2)$, with $\rho=3 \sqrt{3} / 2$. Other points in the outer layer are at distances $\rho=\sqrt{19} / 2$ (points on edges) and $\rho=\sqrt{11} / 2$ (face points).

The Riemann sphere coordinate for any of these points is

$$
z=\frac{y_{1}+i y_{2}}{\rho+y_{3}}
$$

a variant of (2.3). From the inner layer of points, we construct a degree 4 map. The numerator $p(z)$ has roots at $z= \pm \frac{1-i}{\sqrt{3}+1}, \pm \frac{1+i}{\sqrt{3}-1}$ and the denominator $q(z)$ has roots at $z= \pm \frac{1+i}{\sqrt{3}+1}, \pm \frac{1-i}{\sqrt{3}-1}$. This gives

$$
\begin{aligned}
R(z) & =\frac{\left(z+\frac{1-i}{\sqrt{3}+1}\right)\left(z-\frac{1-i}{\sqrt{3}+1}\right)\left(z+\frac{1+i}{\sqrt{3}-1}\right)\left(z-\frac{1+i}{\sqrt{3}-1}\right)}{\left(z+\frac{1+i}{\sqrt{3}+1}\right)\left(z-\frac{1+i}{\sqrt{3}+1}\right)\left(z+\frac{1-i}{\sqrt{3}-1}\right)\left(z-\frac{1-i}{\sqrt{3}-1}\right)} \\
& =\frac{z^{4}+2 \sqrt{3} i z^{2}+1}{z^{4}-2 \sqrt{3} i z^{2}+1}
\end{aligned}
$$

the degree 4 rational map with cubic symmetry, related to the $B=4$ Skyrmion. Cubic symmetry requires that the overall coefficient is of magnitude 1 , and here it is set to 1 .

A similar procedure gives the degree 28 rational map of the outer layer. The numerator 
and denominator are expressed as products of their linear factors. The map is

$$
\begin{aligned}
R(z) & =\frac{\left(z-\frac{3-i}{\sqrt{19}+3}\right)\left(z-\frac{1-3 i}{\sqrt{19}+3}\right)\left(z-\frac{-3+i}{\sqrt{19}+3}\right)\left(z-\frac{-1+3 i}{\sqrt{19}+3}\right)\left(z-\frac{3+3 i}{\sqrt{19}-1}\right)\left(z-\frac{-3+3 i}{\sqrt{19}+1}\right)}{\left(z-\frac{3+i}{\sqrt{19}+3}\right)\left(z-\frac{1+3 i}{\sqrt{19}+3}\right)\left(z-\frac{-3-i}{\sqrt{19}+3}\right)\left(z-\frac{-1-3 i}{\sqrt{19}+3}\right)\left(z-\frac{3+3 i}{\sqrt{19}+1}\right)\left(z-\frac{-3+3 i}{\sqrt{19}-1}\right)} \\
& \times \frac{\left(z-\frac{-3-3 i}{\sqrt{19}-1}\right)\left(z-\frac{3-3 i}{\sqrt{19}+1}\right)\left(z-\frac{3+i}{\sqrt{19}-3}\right)\left(z-\frac{-1-3 i}{\sqrt{19}-3}\right)\left(z-\frac{-3-i}{\sqrt{19}-3}\right)\left(z-\frac{1+3 i}{\sqrt{19}-3}\right)}{\left(z-\frac{-3-3 i}{\sqrt{19}+1}\right)\left(z-\frac{3-3 i}{\sqrt{19}-1}\right)\left(z-\frac{3-i}{\sqrt{19}-3}\right)\left(z-\frac{-1+3 i}{\sqrt{19}-3}\right)\left(z-\frac{-3+i}{\sqrt{19}-3}\right)\left(z-\frac{1-3 i}{\sqrt{19}-3}\right)} \\
& \times \frac{\left(z-\frac{1+i}{\sqrt{11}+3}\right)\left(z-\frac{-1-i}{\sqrt{11}+3}\right)\left(z-\frac{3+i}{\sqrt{11}+1}\right)\left(z-\frac{3-i}{\sqrt{11}-1}\right)\left(z-\frac{1+3 i}{\sqrt{11}+1}\right)\left(z-\frac{-1+3 i}{\sqrt{11}-1}\right)}{\left(z-\frac{1-i}{\sqrt{11}+3}\right)\left(z-\frac{-1+i}{\sqrt{11}+3}\right)\left(z-\frac{3+i}{\sqrt{11}-1}\right)\left(z-\frac{3-i}{\sqrt{11}+1}\right)\left(z-\frac{1+3 i}{\sqrt{11}-1}\right)\left(z-\frac{-1+3 i}{\sqrt{11}+1}\right)} \\
& \times \frac{\left(z-\frac{-3-i}{\sqrt{11}+1}\right)\left(z-\frac{-3+i}{\sqrt{11}-1}\right)\left(z-\frac{-1-3 i}{\sqrt{11}+1}\right)\left(z-\frac{1-3 i}{\sqrt{11}-1}\right)\left(z-\frac{-1+i}{\sqrt{11}-3}\right)\left(z-\frac{1-i}{\sqrt{11}-3}\right)}{\left(z-\frac{-3-i}{\sqrt{11}-1}\right)\left(z-\frac{-3+i}{\sqrt{11}+1}\right)\left(z-\frac{-1-3 i}{\sqrt{11}-1}\right)\left(z-\frac{1-3 i}{\sqrt{11}+1}\right)\left(z-\frac{1+i}{\sqrt{11}-3}\right)\left(z-\frac{-1-i}{\sqrt{11}-3}\right)} \\
& \times \frac{\left(z-\frac{-1-i}{\sqrt{3}+1}\right)\left(z-\frac{1+i}{\sqrt{3}+1}\right)\left(z-\frac{-1+i}{\sqrt{3}-1}\right)\left(z-\frac{1-i}{\sqrt{3}-1}\right)}{\left(z-\frac{1-i}{\sqrt{3}+1}\right)\left(z-\frac{-1+i}{\sqrt{3}+1}\right)\left(z-\frac{1+i}{\sqrt{3}-1}\right)\left(z-\frac{-1-i}{\sqrt{3}-1}\right)} .
\end{aligned}
$$

Cubic symmetry and matching to the inner layer map fixes the overall coefficient to be 1. The linear factors could be multiplied out, giving a map of the structure discussed in [10]. However, there is little reason to do this: the linear factor representation makes it easier to check symmetry and to recall the coordinates of the half-Skyrmions. It also avoids problems with overflowing numerics.

This degree 28 rational map is not optimal energetically, but it is close to optimal because the zeros and poles are approximately evenly spread over the Riemann sphere, and they are rather well separated from each other. Using these maps of degrees 4 and 28 in the double rational map ansatz as initial data, with a simple, piecewise-linear radial profile function, we successfully recover the cubically symmetric $B=32$ Skyrmion using the numerical relaxation algorithm developed in [19].

The profile functions we have used are shown in Figure 4. The profile functions coloured black, red and blue are used in the single, double and triple rational map ansatz, respectively. Since $f(r)$ is an integer multiple of $\pi$ in finite intervals of $r$, the Skyrme field $U$ initially takes the value $\pm \boldsymbol{I}$ in spherical shells of finite thickness.

\subsection{Exploring $B=24-31$ solutions using the corner cutting method}

The idea of cutting single Skyrmions from the corners of the $B=32$ Skyrmion was proposed in [16] to generate new solutions with baryon numbers from $B=31$ down to $B=24$. The corner cutting procedure is done on the degree 28 rational map (3.3); it is best understood using the geometrical approach to rational maps presented above. The inner degree 4 map is unchanged. 


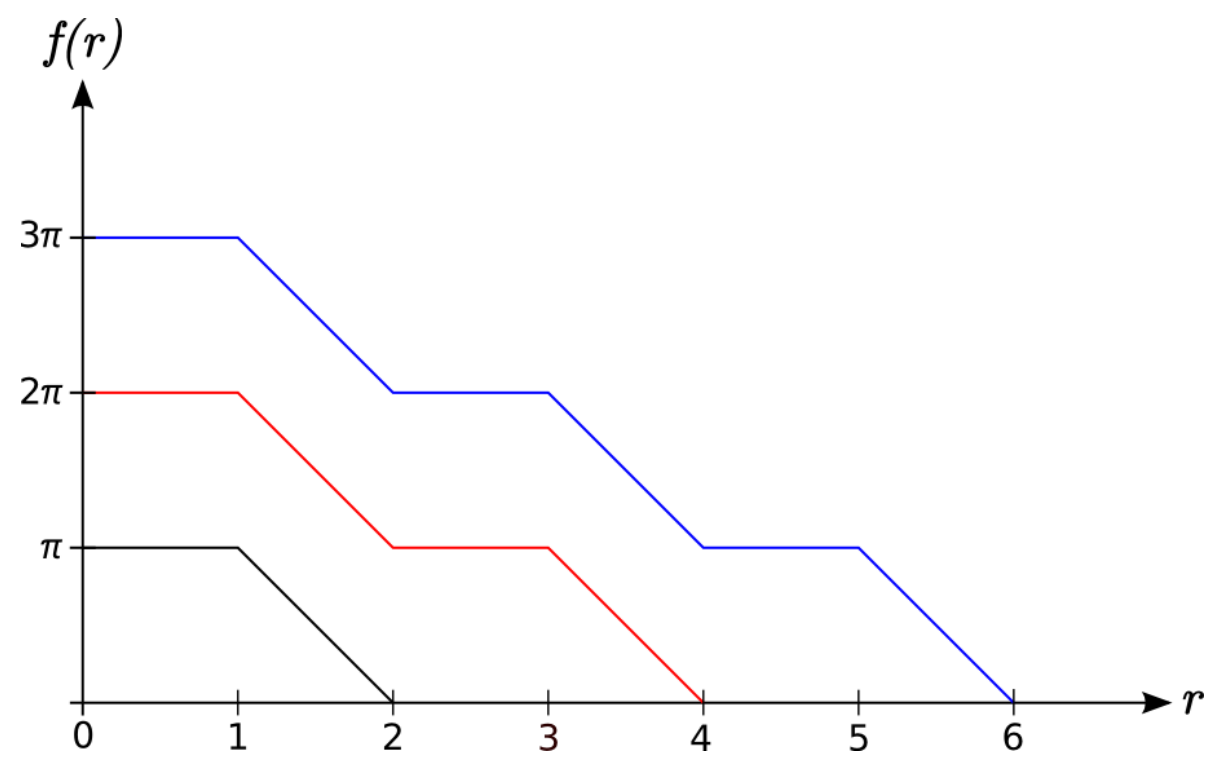

Figure 4: Profile functions $f(r)$ used for initial data

The simplest way to remove a Skyrmion from a rational map is to decrease its degree by one by merging a zero and a pole. Linear factors cancel in the numerator and denominator. Say $R(z)$ has a zero at $a$ and a pole at $b$. Then eliminating one Skyrmion is done by setting $a=b$ :

$$
\left.R(z)\right|_{a=b}=\left.\frac{p(z)}{q(z)}\right|_{a=b}=\left.\frac{(z-a) P(z)}{(z-b) Q(z)}\right|_{a=b}=\frac{P(z)}{Q(z)},
$$

where $P(z)$ and $Q(z)$ are the remaining parts of the polynomials $p(z)$ and $q(z)$.

We could use this method on one of the corner poles (or zeros) of the map (3.3) and one of its neighbouring zeros (or poles), but this destroys all the symmetry, which is not desirable. Rather, to preserve as much symmetry as possible, the three neighbouring zeros are moved simultaneously towards a corner pole. The pole cancels against one of the zeros, leaving a double zero at the corner after cancellation (see Figure 5). Notice that what had been a black corner becomes a white one with a hole. The $O_{h}$ symmetry is broken down to $C_{3 v}$. After numerical relaxation, this method gives a new stable Skyrmion with $B=31$. This corner-cutting procedure can be repeated up to eight times. At each corner, three poles are merged with one zero, or three zeros with one pole, and the result is either a double pole or a double zero. These persist in the Skyrmion solutions as holes in the baryon density at corners that have been cut.

We have obtained solutions from $B=24$ to $B=31$ using this method. The $B=32$ Skyrmion looks like eight copies of the cubic $B=4$ Skyrmion. In the $B=31$ Skyrmion, one of the $B=4$ cubes becomes a slightly deformed $B=3$ tetrahedral Skyrmion. The remaining seven hardly change, because the interactions between Skyrmions at the corners are weak. When this corner cutting is repeated, further $B=4$ cubes are replaced by $B=3$ tetrahedra. In order to preserve as much symmetry as possible, we cut the 

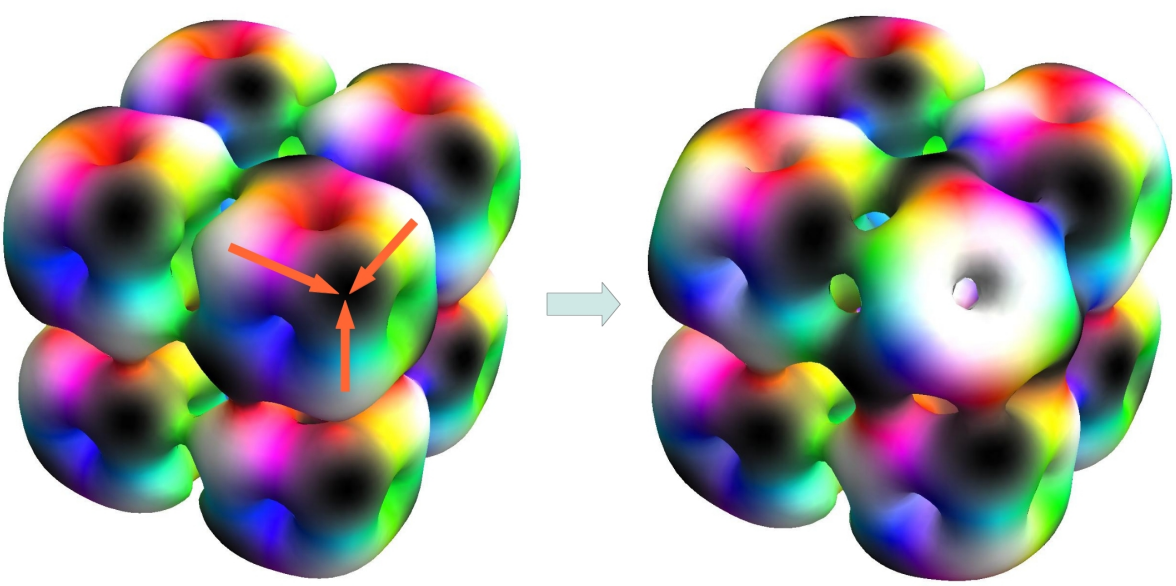

Figure 5: Moving three zeros (white) towards a pole (black), to produce the $B=31$ Skyrmion

corners as follows. For $B=30$, a pair of diagonally opposite corners are cut and $D_{3 d}$ symmetry remains. For $B=29$, we need to remove three corners and cutting diagonally opposite corners is no longer a desirable option. Instead, three corners, with each pair face-diagonally opposite, are cut. For $B=28$, four corners forming a tetrahedron are removed and $T_{d}$ symmetry remains. One further corner is removed to generate the $B=27$ Skyrmion. For $B=26$, just two diagonally opposite corners are left uncut. The field relaxation is initially similar to the higher $B$ cases, but now there is a change. The

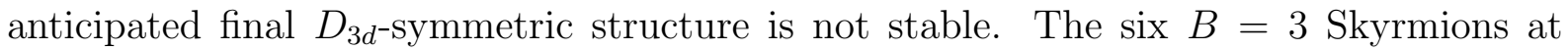
the cut corners move towards the plane mid-way between the uncut corners. Then the $B=4$ cubes move in opposite ways and join up, one each, with three of the six $B=3$ Skyrmions forming a structure consisting of two similar $B=13$ clusters (see Figure 6a). The $B=13$ cluster has only one reflection symmetry and smaller Skyrmion clusters could not be identified clearly within it.

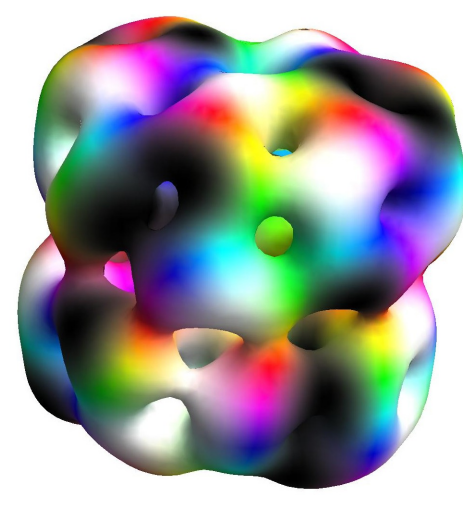

(a) $B=26$

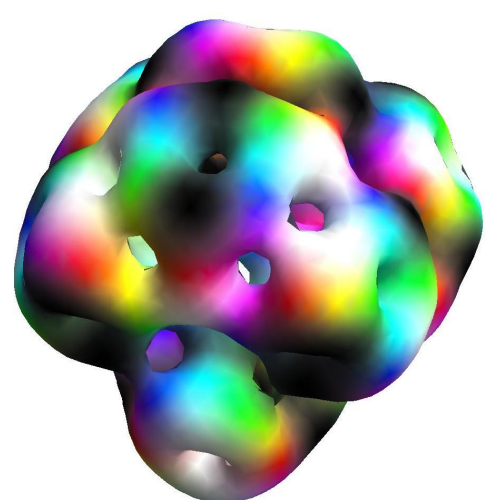

(b) $B=25$

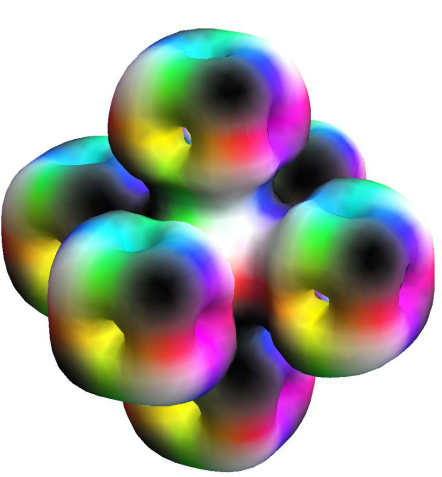

(c) $B=24$

Figure 6: Skyrmions from cutting corners 
For $B=25$, seven corners are cut initially. However, the relaxed $B=25$ Skyrmion again does not have the shape expected from corner cutting (see Figure 6b). The relaxed Skyrmion only has a $C_{1 h}$ reflection symmetry, but we can still vaguely identify cubic $B=4$ Skyrmions and tetrahedral $B=3$ Skyrmions within the cluster.

$B=24$ is the most interesting case. Cutting all eight corners from the $B=32$ Skyrmion produces a cubically symmetric solution that is best thought of as $\operatorname{six} B=4$ cubes at the vertices of the dual octahedron of the $B=32$ cube, rather than eight $B=3$ Skyrmions at the vertices of the original cube. Some half-Skyrmions of the $B=4$ cubes are acquired from the $B=3$ Skyrmions (see Figure 6c, and note the fate of the three black regions near a white corner).

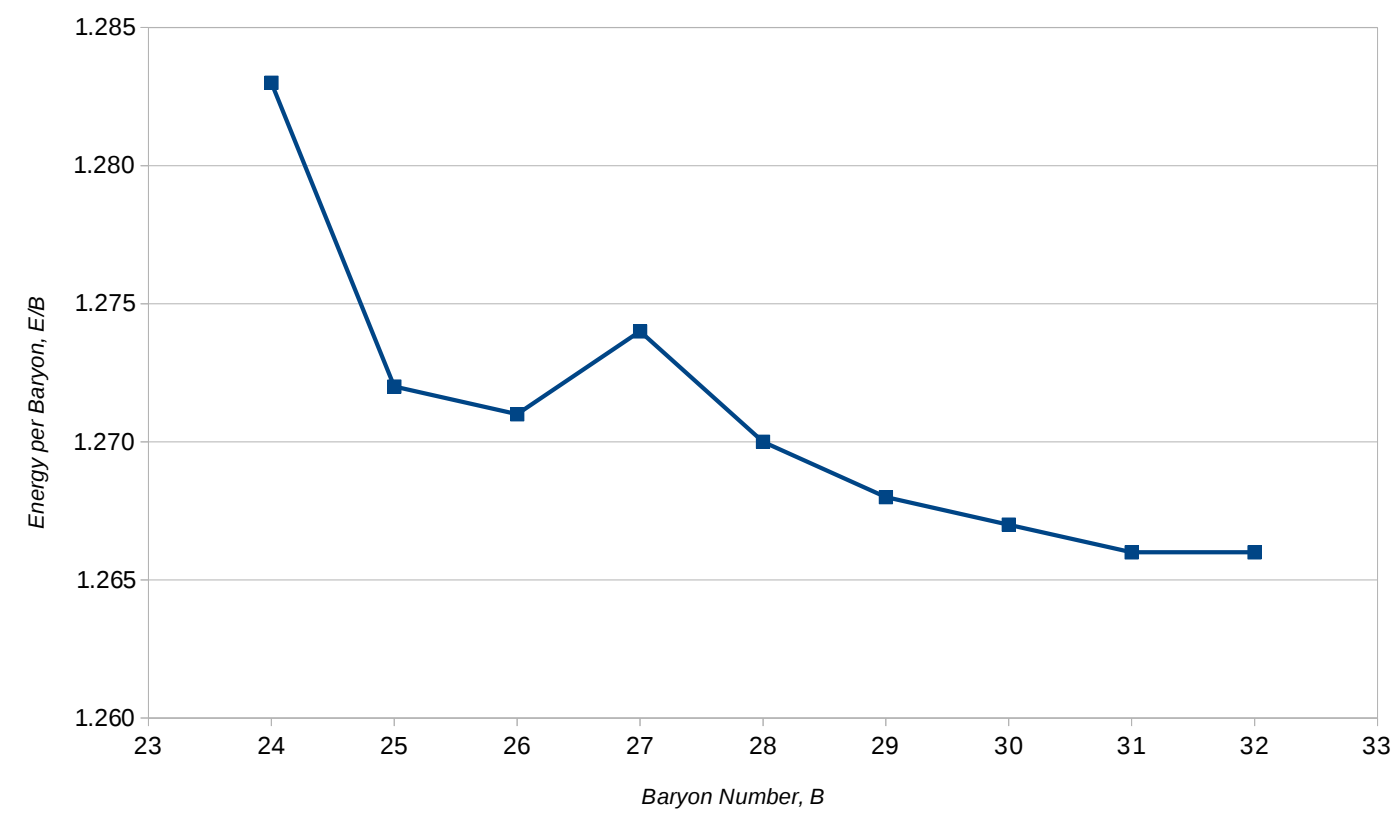

Figure 7: Energy per baryon for $B=24$ to $B=32$

Figure 7 shows the energy per baryon, $E / B$, of the Skyrmions found using this corner cutting technique. (They are also tabulated in Table 1.) For $B=27$ to $B=31$, the structures of the Skyrmions are pretty close to that of the parent $B=32$ solution. The energy per baryon varies smoothly in this range. For solutions from $B=24$ to $B=26$, however, the energy is lower than the extrapolation of this smooth behaviour. This shows that corner cutting is only a first step and further relaxation occurs due to the interaction between corners. 


\section{$3.3 \quad B=100-108$ solutions}

The next in the sequence of $B=4 n^{3}$ cubic Skyrmions is $B=108$. Using a $6 \times 6 \times 6$ cubic grid, we have constructed a degree 76 outer rational map (analogous to (3.3) but too long to write out). This is combined with the degree 28 and degree 4 maps from the $B=32$ Skyrmion, as middle and inner maps. A suitable profile function $f(r)$ running from $3 \pi$ to 0 is used for the initial configuration (see Figure 4). After relaxation, the stable Skyrmion shown in Figure 8 a was obtained. It has the familiar structure of touching $B=4$ subunits all with the same orientation.

The next step is to remove single Skyrmions from the corners. This was done, as in the $B=32$ case, by merging three zeros (poles) of the outer map with a pole (zero) at each corner. This procedure generates Skyrmions with all baryon numbers from 107 down to 100. The cubic structure is locally retained, except that the $B=4$ cubes at the corners are replaced by $B=3$ tetrahedra. Recall that for the $B=32$ Skyrmion there was little structural change observed until six or more corner Skyrmions were removed. We find the $B=108$ Skyrmion to be more stable to corner cutting as the corners are further away from each other. Removing first four, and then all eight corners gives the tetrahedral $B=104$ Skyrmion and cubic $B=100$ Skyrmion shown in Figures $8 \mathrm{~b}$ and $8 \mathrm{c}$.

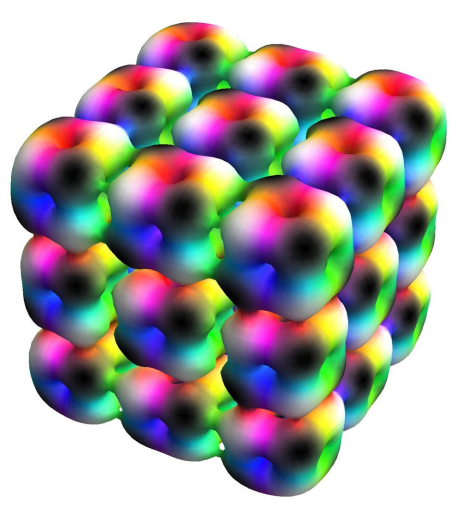

(a) $B=108$ Skyrmion

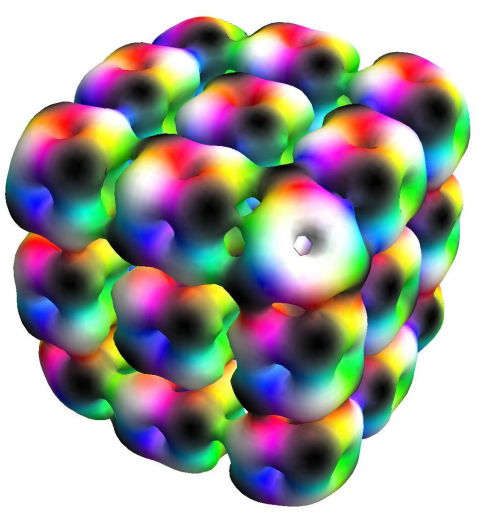

(b) $B=104$ Skyrmion with 4 corners cut

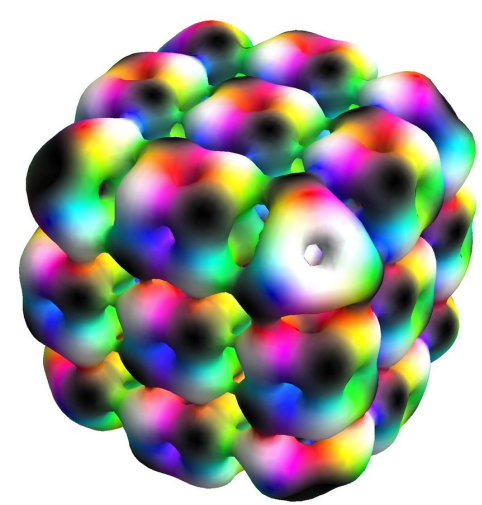

(c) $B=100$ Skyrmion with 8 corners cut

Figure 8: Skyrmions from triple-layer rational maps

\section{Rational maps with $O_{h}$ and $T_{d}$ symmetry}

\subsection{Klein polynomials and the degree 28 map}

The Skyrme crystal is cubically symmetric so the symmetries of rational maps constructed from the crystal are related to $O_{h}$. $O_{h}$ itself and its subgroup $T_{d}$, the full symmetry groups 
of the cube and tetrahedron, are of particular importance.

To study the interplay of the geometrical method and symmetry, it is useful to recall the tetrahedrally symmetric Klein polynomials [20] and to express our degree 28 map (3.3) in terms of these. In our preferred orientation, the tetrahedral vertex and face polynomials $p_{+}(z)$ and $p_{-}(z)$ are

$$
\begin{aligned}
& p_{+}(z)=z^{4}+2 \sqrt{3} i z^{2}+1, \\
& p_{-}(z)=z^{4}-2 \sqrt{3} i z^{2}+1 .
\end{aligned}
$$

The ratio of these degree 4 polynomials is the rational map $R(z)=p_{+}(z) / p_{-}(z)$ of the $B=4$ Skyrmion. This is not just tetrahedrally, but cubically symmetric. The extra symmetry under a $90^{\circ}$ rotation sends $z$ to $i z$, and hence $R(z)$ to $1 / R(z)$.

$p_{+} / p_{-}$turns out to be the most important ingredient in the geometrically generated rational maps. For example, the 56 points in the outer layer of the $4 \times 4 \times 4$ cubic grid shown in Figure 3 can be separated into three subsets: those on the face interiors, those on the edges and those on the vertices (see Figure 91). Each subset has cubic symmetry.

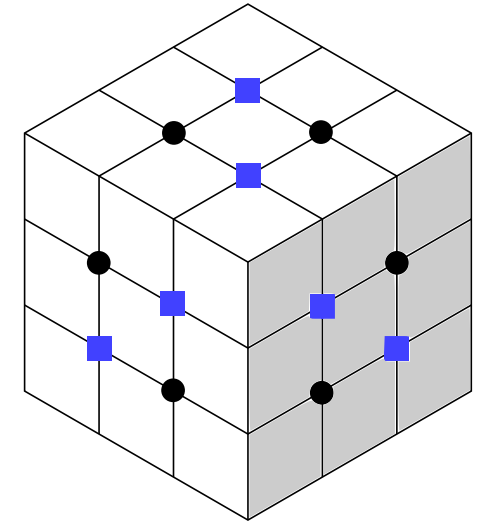

(a) Points on face interiors

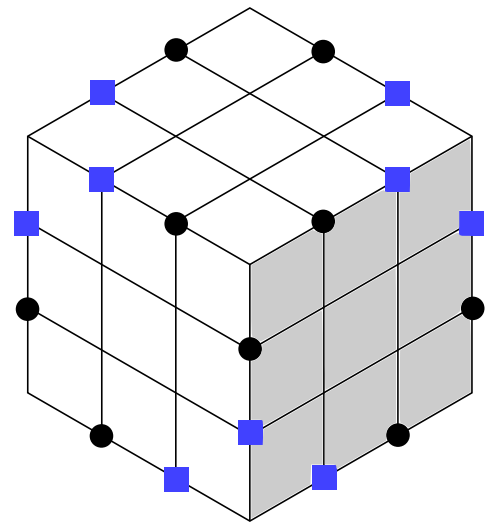

(b) Points on edges

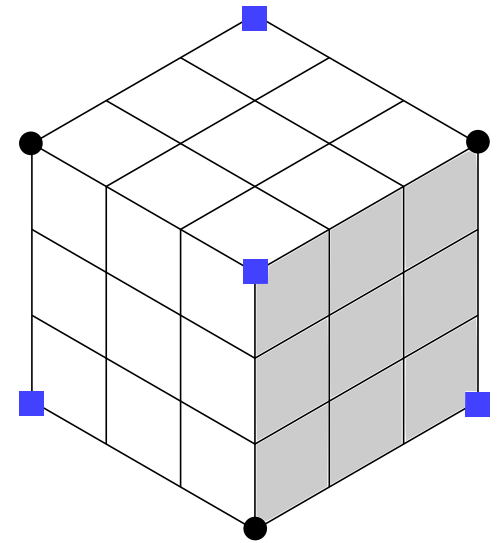

(c) Points on vertices

Figure 9: Points of the $4 \times 4 \times 4$ cubic grid

It is convenient to denote the rational maps constructed from these subsets by $R_{F}$, $R_{E}$ and $R_{V}$. They are of degrees 12, 12 and 4, respectively, and $R_{V}$ is the familiar cubic degree 4 map 3.2 . $R_{F}$ and $R_{E}$ are (projectively) linearly related to $R_{V}{ }^{3}=p_{+}^{3} / p_{-}^{3}$ by

$$
R_{F}=\frac{c_{1} p_{+}{ }^{3}+p_{-}{ }^{3}}{p_{+}{ }^{3}+c_{1} p_{-}{ }^{3}}, \quad R_{E}=\frac{c_{2} p_{+}{ }^{3}+p_{-}{ }^{3}}{p_{+}{ }^{3}+c_{2} p_{-}{ }^{3}}
$$

with $c_{1}=-2.873$ and $c_{2}=0.178$. (Both $c_{1}$ and $c_{2}$ can be expressed in analytical form, but this is messy and provides no further insight.) Relations 4.2 can be understood by noting that $O_{h}$ symmetry does not uniquely fix 24 points on the Riemann sphere. There 
is a family with one real parameter $c$ having this symmetry,

$$
R_{(c)}=\frac{c p_{+}{ }^{3}+p_{-}{ }^{3}}{p_{+}{ }^{3}+c p_{-}{ }^{3}} .
$$

Starting from $c=-1$, the numerator and the denominator of the rational map $R_{(c)}$ cancel completely and the map takes a constant value of -1 which corresponds to the two zeros and the two poles on each face in Figure 9a coinciding at the centre of the face. As $c$ varies from -1 to $-\infty$, the points move from the face centres along face diagonals to the vertices; the rational map $R_{F}$ is a special case. At $c=-\infty$, three zeros or three poles coincide at the vertices and $R_{V}{ }^{3}$ is recovered. We next identify $c=-\infty$ and $c=\infty$ as they give the same rational map. When $c$ varies from $\infty$ to 1 , the points move along the edges and the rational map changes from $R_{V}{ }^{3}$ to a constant value of 1 where the zeros and poles coincide at the middle of the edges. The range of the parameter $c$ has now covered the lower half circle of Figure 10. The rational map $R_{(c)}$ behaves similarly for $c$ taking values on the upper half circle but with the numerator and denominator interchanged (zeros become poles and vice versa). The rational map $R_{E}$ is a special case in this range.

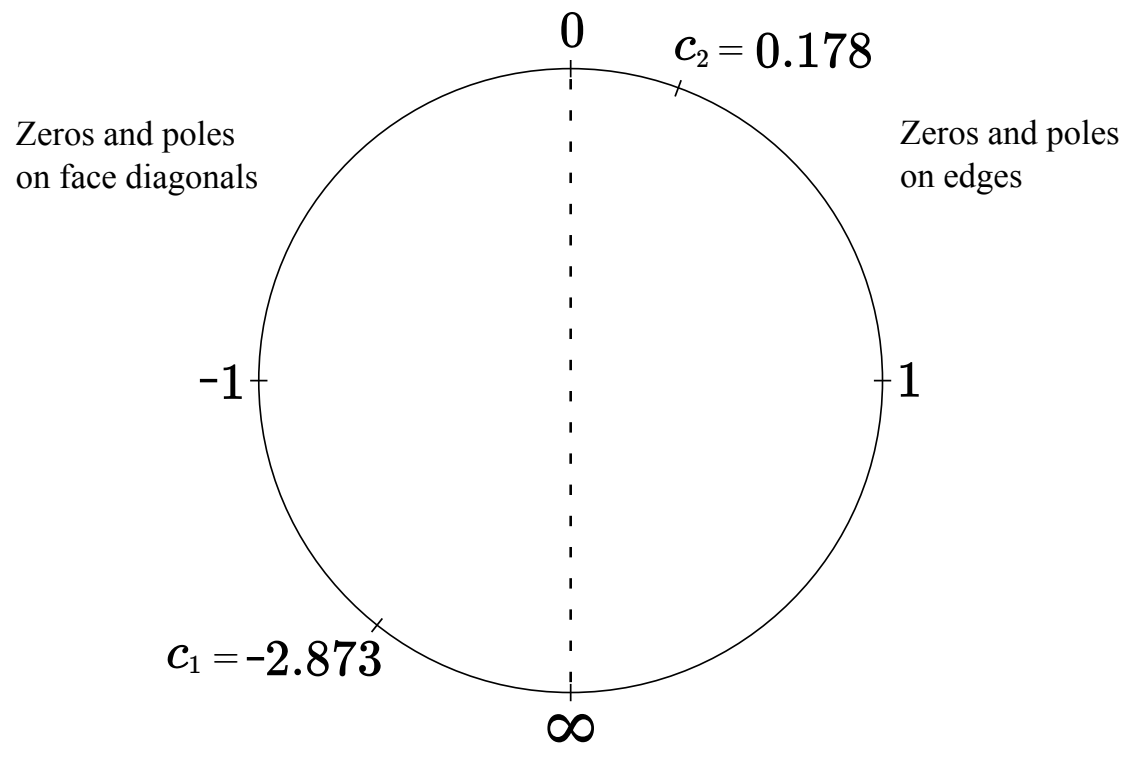

Figure 10: Range of $c$

We now see that

$$
\begin{aligned}
R_{V} \times R_{F} \times R_{E} & =\frac{p_{+}}{p_{-}}\left(\frac{c_{1} p_{+}^{3}+p_{-}^{3}}{p_{+}^{3}+c_{1} p_{-}^{3}}\right)\left(\frac{c_{2} p_{+}^{3}+p_{-}^{3}}{p_{+}^{3}+c_{2} p_{-}^{3}}\right) \\
& =\frac{p_{+}}{p_{-}}\left(\frac{c_{1} c_{2} p_{+}^{6}+\left(c_{1}+c_{2}\right) p_{+}^{3} p_{-}^{3}+p_{-}^{6}}{p_{+}^{6}+\left(c_{1}+c_{2}\right) p_{+}^{3} p_{-}^{3}+c_{1} c_{2} p_{-}^{6}}\right),
\end{aligned}
$$

and this can be compared with the degree 28 map (modulo sign flips) constructed by the optimisation method,

$$
R=\frac{p_{+}}{p_{-}}\left(\frac{-C p_{+}^{6}-D p_{+}^{3} p_{-}^{3}+p_{-}^{6}}{p_{+}^{6}-D p_{+}^{3} p_{-}^{3}-C p_{-}^{6}}\right)
$$


The values of $C$ and $D$ calculated from $c_{1}$ and $c_{2}$ are 0.51 and 2.70, respectively, which are moderately close to the values $C=0.33$ and $D=1.64$ in [10].

\section{2 $B=20$ Skyrmions}

We have also used the rational maps $R_{F}, R_{E}$ and $R_{V}$ to seek the $B=20$ Skyrmion, which for massive pions was not previously firmly established. The $\alpha$-particle model of nuclei suggests that the $B=20$ Skyrmion is formed from five $B=4$ Skyrmions. A solution of this type was found earlier [10], but was not of the expected triangular bi-pyramid shape, had little symmetry and was probably not of minimal energy.

Two useful rational maps are $R=R_{V} \times R_{F}$ and $R=R_{V} \times R_{E}$. Their zeros and poles are shown in Figure 11. Each has degree 16 and can be used as an outer map. The inner map is the cubically symmetric degree 4 map (3.2). Using these pairs of maps in the double rational map ansatz, and relaxing, gives two candidate $B=20$ Skyrmions, but neither has a bi-pyramid shape. The cubic symmetry is also not rigorously enforced by the numerics, and it ends up broken. The first Skyrmion has $T_{d}$ symmetry, and the second only $D_{2 h}$ symmetry.

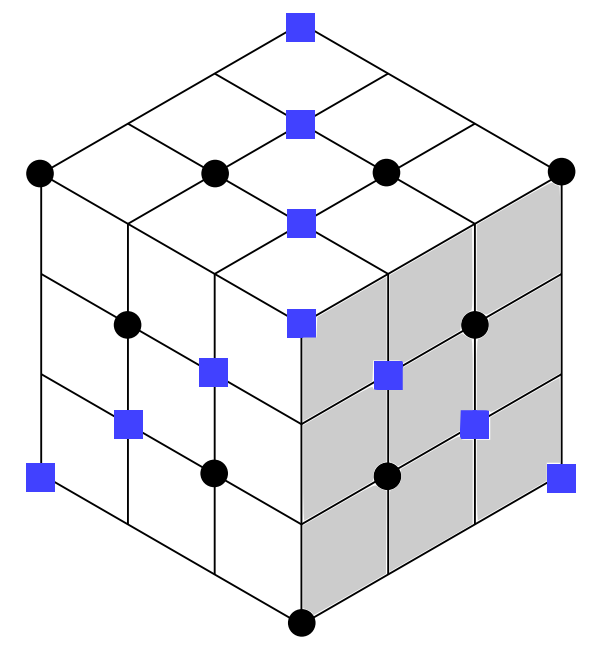

(a) Points generating the $T_{d^{-}}$ symmetric Skyrmion

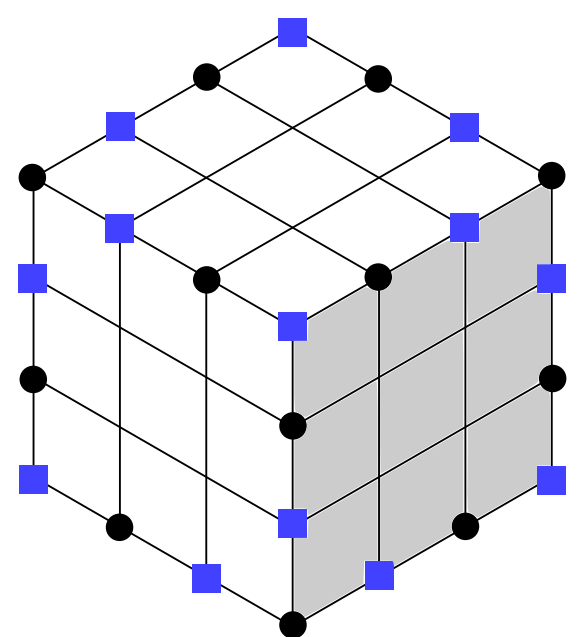

(b) Points generating the $D_{2 h^{-}}$ symmetric Skyrmion

Figure 11: Grid points used for $B=20$

The $T_{d}$-symmetric solution has an energy per baryon $E / B=1.277$. It can be interpreted as four slightly distorted $B=4$ cubes at the vertices of a tetrahedron and four $B=1$ Skyrmions at the face centres of the tetrahedron (see Figure 12a). Each $B=1$ Skyrmion is oriented such that the colour equator $\left(\hat{\boldsymbol{\pi}}_{1}-\hat{\boldsymbol{\pi}}_{2}\right.$ plane) is parallel to the face of the tetrahedron containing the Skyrmion. Its primary colours match the colours of the faces of the $B=4$ cubes that it touches. As a result, the $B=1$ and $B=4$ Skyrmions 
are all attracting. The $D_{2 h}$-symmetric solution has slightly lower energy per baryon, $E / B=1.274$, and appears to be the lowest energy solution for $B=20$. The Skyrmion consists of two loosely touching clusters, each in the form of the known $B=10$ Skyrmion (see Figure 12b) [5].

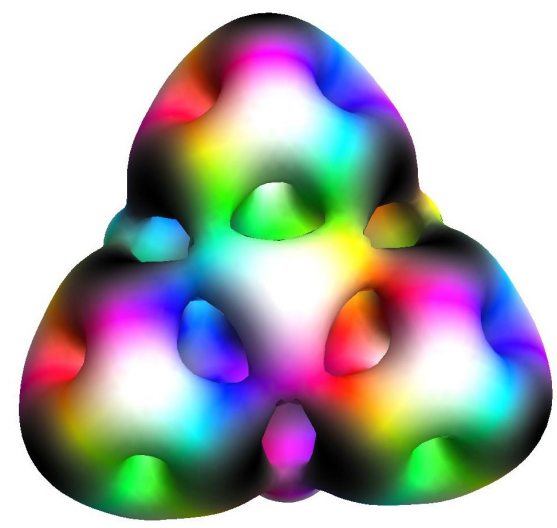

(a) $B=20$ Skyrmion with $T_{d}$ symmetry

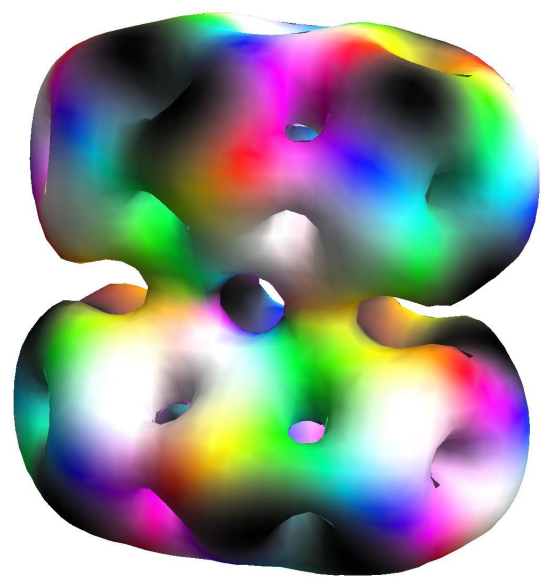

(b) $B=20$ Skyrmion formed by stacking two $B=10$ Skyrmions

Figure 12: $B=20$ Skyrmions

The $B=10$ Skyrmion itself resembles two $B=4$ cubes bound together by two $B=1$ Skyrmions. Figure 13 shows the top view of the $B=20$ Skyrmion which looks the same as the $B=10$ Skyrmion in [5]. The $B=10$ Skyrmion can be reproduced using the geometric method, and has $E / B=1.280$. As expected, the $B=20$ Skyrmion has slightly lower energy than two well-separated $B=10$ Skyrmions.

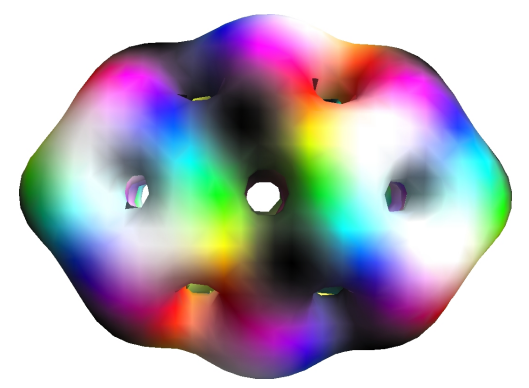

Figure 13: Top view of $B=20$ with $D_{2 h}$ symmetry

Since the energy difference between the $T_{d}$-symmetric and $D_{2 h}$-symmetric solutions is only about $0.2 \%$, it is reasonable to ask how similar the solutions are. The relaxation of the Skyrme field was monitored. It appears that for the $T_{d}$-symmetric Skyrmion, it is energetically favourable to rotate one of the edges of the tetrahedron relative to the 
opposite one by $90^{\circ}$. If a deformation is introduced breaking the tetrahedral symmetry, the field relaxes to the $D_{2 h}$-symmetric solution.

To obtain the $T_{d}$-symmetric solution, it is actually preferable to start with an outer rational map with $T_{d}$ rather than $O_{h}$ symmetry. The initial field configuration will likely be closer to the Skyrmion and convergence faster. A suitable degree 16 outer map has been constructed using the set of points shown in Figure 14. The degree 4 inner map is unchanged. This combination builds in the four $B=4$ Skyrmions and the four $B=1$ Skyrmions rather effectively.

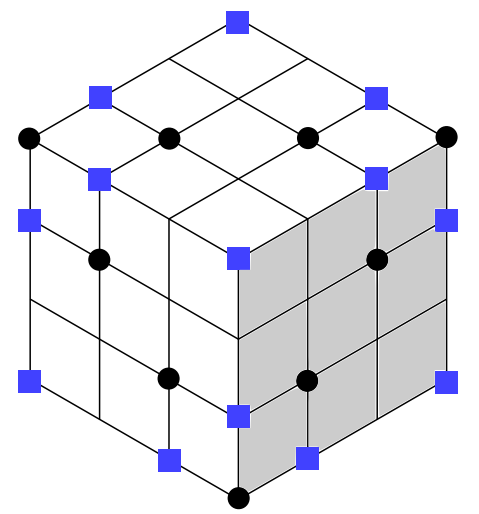

Figure 14: Zeros and poles with $T_{d}$ Symmetry

This rational map with $T_{d}$ symmetry can again be written in terms of $p_{+}$and $p_{-}$, and is

$$
R=\left(\frac{1+c_{2}}{1+c_{1}}\right) \frac{p_{+}}{p_{-}}\left(\frac{c_{1} p_{+}^{3}+p_{-}^{3}}{p_{+}^{3}+c_{2} p_{-}^{3}}\right),
$$

where $c_{1}$ and $c_{2}$ are as in 4.2 . It is a bit more complicated than an $O_{h}$-symmetric map.

\section{$5 \quad$ Skyrmions obtained by other methods}

It is established that Skyrmions often cluster into $B=4$ units, even when this is not imposed in the first place. One may therefore take the $B=4$ Skyrmion as a basic building block and assemble larger Skyrmions from it, using the product ansatz followed by numerical relaxation. This has been done previously for $B=8, B=12$ and $B=32$. We have now found solutions for $B=24$ in this way.

The cubic $B=4$ Skyrmion (Figure 2p has alternating white and black half-Skyrmions at the corners. In the standard orientation defined by the rational map (3.2), its face colours are red, green and blue, and opposite faces have the same colour. This means that two cubes in the same orientation, face-to-face, will have matching face colours, but the corner colours will not match. This is the situation in the Skyrme crystal, where the 

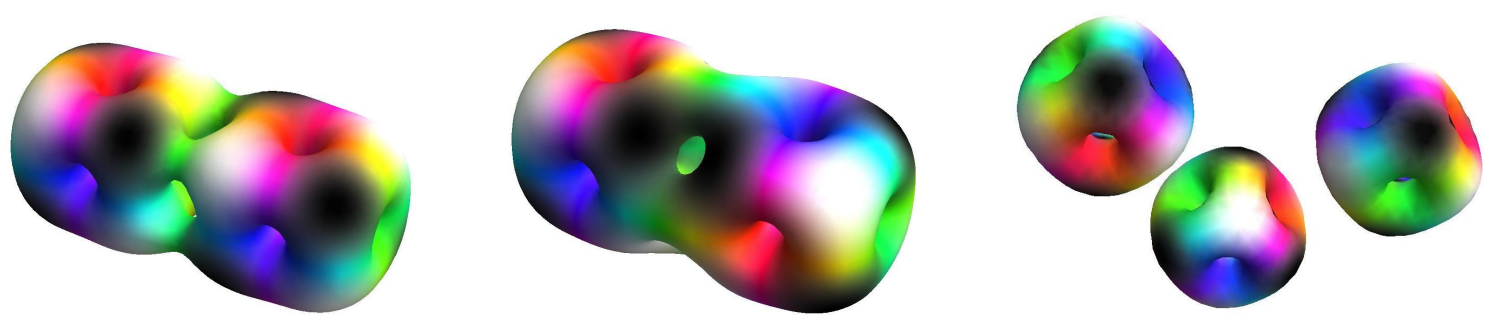

Figure 15: Left: Two $B=4$ cubes bound together into a $B=8$ solution. Middle: The same, but one cube is rotated around the axis of separation; this is the lower energy solution. Right: Three cubes in an L-shape.

$B=4$ cubes are all oriented the same way, and also in crystal chunks, such as the $B=32$ and $B=108$ Skyrmions.

Given just two cubes, we can twist one of them by $90^{\circ}$ around the axis of separation (see Figure 15). Then both the face and corner colours match, although the edge colours do not. This is the minimal energy configuration in the $B=8$ sector for the Skyrme model with massive pions. However, it is not possible to construct a crystal with these twists: After arranging three $B=4$ cubes in an L-shape with $90^{\circ}$ twists, the neighbouring empty space will be bounded by two faces of the same colour, so a further cube cannot be inserted with low energy to make a $B=16$ square (see Figure 15 , right).

One can get a $B=24$ solution, as a crystal chunk, by removing two $B=4$ cubes from opposite corners of the $B=32$ Skyrmion. This results in a non-planar ring of six $B=4$ cubes, all with the same orientation (Figure 16a). However, in this case, we can do better. Because of the two missing corner cubes, we can re-orient the remaining six cubes so that each neighbouring pair has a $90^{\circ}$ relative twist around its separation axis (Figure 16b). This results in a lower energy. These two $B=24$ solutions have $E / B=1.273$ and $E / B=1.269$, respectively, and the latter may be the true $B=24$ Skyrmion of minimal energy.

\section{Conclusions}

In this paper we have presented several new Skyrmions for pion mass parameter $m=1$, with baryon numbers as high as $B=108$. We have used insight obtained from the infinite Skyrme crystal to develop a geometrical method to construct multi-layer rational map ansätze for the Skyrme field. This gives fairly simple algebraic expressions for initial data, which have then been relaxed numerically to obtain Skyrmions which are either global or local minima of the energy.

The cubic $B=32$ Skyrmion, previously known, was constructed afresh using a double rational map ansatz. A range of novel Skyrmions from $B=24$ up to $B=31$, related to 


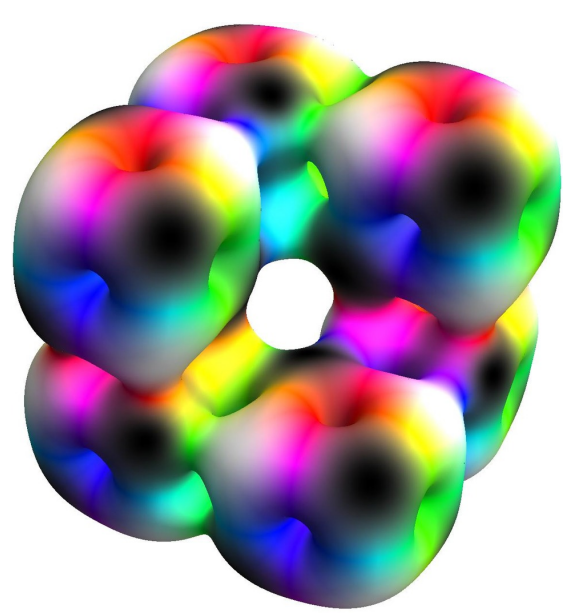

(a) $B=24$ Skyrmion as crystal chunk

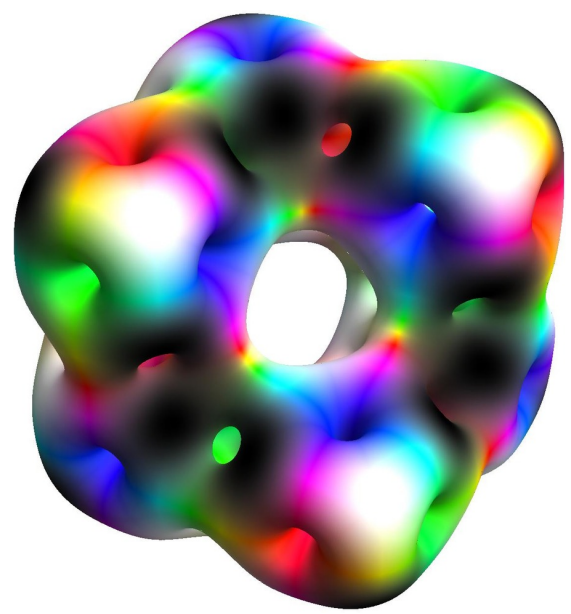

(b) $B=24$ Skyrmion with twisted cubes

Figure 16: $B=24$ Skyrmions

this $B=32$ Skyrmion, were then obtained by modifying the outer rational map, so as to remove one unit of baryon number from any of the eight corners. Further modifications of the rational map led to new Skyrmions with $B=20$.

For the first time, a three-layer rational map ansatz has been used. This successfully generated the cubic $B=108$ Skyrmion, the first Skyrmion with baryon number $B>100$ to be found. Corner cutting gave solutions in the range $B=100$ to $B=107$.

Using another approach, we have found what may be the lowest energy Skyrmion with $B=24$. This was constructed by gluing together, with twists, six $B=4$ cubic Skyrmions into a ring, and relaxing.

The next step would be to consider quantisation of the newly found Skyrmions in order to get further nuclear spectra from the Skyrme model. Nuclear spectra calculations are currently limited to baryon numbers no higher than 16 [7, 21]. 


\begin{tabular}{|c|c|c|c|c|}
\hline Baryon Number $B$ & Energy $E$ & $E / B$ & Symmetry & Comment \\
\hline 1 & 1.465 & 1.465 & $O(3)$ & Hedgehog \\
\hline 2 & 2.77 & 1.385 & $D_{\infty h}$ & Toroid \\
\hline 3 & 4.02 & 1.340 & $T_{d}$ & Tetrahedral Skyrmion \\
\hline 4 & 5.18 & 1.295 & $O_{h}$ & Cubic Skyrmion \\
\hline 8 & $\begin{array}{l}10.25 \\
10.28\end{array}$ & $\begin{array}{l}1.281 \\
1.285\end{array}$ & $\begin{array}{l}D_{4 h} \\
D_{4 h}\end{array}$ & $\begin{array}{l}\text { Two } B=4 \text { cubes with } 90^{\circ} \text { twist } \\
\text { Two } B=4 \text { cubes without twist }\end{array}$ \\
\hline 10 & 12.80 & 1.280 & $D_{2 h}$ & \\
\hline 20 & $\begin{array}{l}25.47 \\
25.53\end{array}$ & $\begin{array}{l}1.274 \\
1.277\end{array}$ & $\begin{array}{c}D_{2 h} \\
T_{d}\end{array}$ & Two $B=10$ clusters \\
\hline 24 & $\begin{array}{l}30.47 \\
30.57 \\
30.80\end{array}$ & $\begin{array}{l}1.269 \\
1.273 \\
1.283\end{array}$ & $\begin{array}{l}D_{3 d} \\
D_{3 d} \\
O_{h}\end{array}$ & $\begin{array}{l}\text { Six } B=4 \text { cubes with twists } \\
\text { Six } B=4 \text { cubes without twists } \\
\text { Octahedral cluster of } B=4 \text { cubes } \\
\text { dual to } B=32 \text { cubic Skyrmion }\end{array}$ \\
\hline 25 & 31.79 & 1.272 & $C_{1 h}$ & $\begin{array}{l}\text { A cluster of } B=4 \text { and } B=3 \\
\text { Skyrmions }\end{array}$ \\
\hline 26 & 33.05 & 1.271 & $C_{2 h}$ & Two $B=13$ clusters \\
\hline 27 & 34.39 & 1.274 & $C_{3 v}$ & \\
\hline 28 & 35.57 & 1.270 & $T_{d}$ & \\
\hline 29 & 36.78 & 1.268 & $C_{3 v}$ & \\
\hline 30 & 38.00 & 1.267 & $D_{3 d}$ & \\
\hline 31 & 39.25 & 1.266 & $C_{3 v}$ & \\
\hline 32 & 40.51 & 1.266 & $O_{h}$ & $\begin{array}{l}\text { Cubic cluster of eight } B=4 \\
\text { cubes }\end{array}$ \\
\hline 100 & 125.68 & 1.257 & $O_{h}$ & \\
\hline 101 & 126.86 & 1.256 & $C_{3 v}$ & \\
\hline 102 & 128.05 & 1.255 & $D_{3 d}$ & \\
\hline 103 & 129.26 & 1.255 & $C_{3 v}$ & \\
\hline 104 & 130.47 & 1.255 & $T_{d}$ & \\
\hline 105 & 131.71 & 1.254 & $C_{3 v}$ & \\
\hline 106 & 132.95 & 1.254 & $D_{3 d}$ & \\
\hline 107 & 134.21 & 1.254 & $C_{3 v}$ & \\
\hline 108 & 135.47 & 1.254 & $O_{h}$ & Cubic cluster of $27 B=4$ cubes \\
\hline$\infty$ & - & 1.238 & $O_{h}$ & Skyrme Crystal [19] \\
\hline
\end{tabular}

Table 1: Energies and symmetries of Skyrmions (for $m=1$ ). The energy $E$ is accurate to \pm 0.01 


\section{Acknowledgements}

D.T.J Feist is supported by the Gates Cambridge Trust and EPSRC. P.H.C. Lau is supported by Trinity College, Cambridge.

\section{A Appendix: Numerical methods}

All of the Skyrme field relaxations were done with the numerical methods developed in [19], with some extensions. The numerics are based on an $(N+1)^{3}$ cubic grid with lattice constant $h$, on which the Skyrme energy from equation (1.3) was discretised using sixthorder finite differences. This particular discretisation order was chosen because it gives the highest precision per computation time.

To find the minimum of the discretised energy, a nonlinear conjugate gradient (NLCG) method was chosen [22]. NLCG can be seen as a geometrically enhanced version of gradient descent. The latter is a very slow method, in particular when the Hessian is of poor condition. NLCG converges much faster.

Another way to find minima of the energy is to compute the time development using a version of the dynamical Skyrme field equations. The advantage is that the field will accelerate towards an energy minimum, but the disadvantage is that it will then overshoot if no measures are taken. The simplest remedy is to take out kinetic energy by introducing a friction term, but this eliminates the real advantage over gradient descent. A more advanced method is to take out all kinetic energy whenever the potential energy is increasing.

One may ignore nonlinear terms with time derivatives in the Skyrme field equation in this process, which gives a simpler, if not quite correct time development. This does not matter, as long as the Lagrangian includes the correct static energy. The time development will still converge to minima of the energy [23]. This method gives rapid convergence and has been used to compute many Skyrmions [24].

Starting close to an energy minimum, the NLCG method converges sufficiently rapidly, in our experience. From further away, time development is often faster at producing the required structural changes to the field. This is expected, because far away from a quadratic minimum, NLCG is not much better than simple gradient descent methods, and these tend to zig-zag.

All numerical methods were implemented in $\mathrm{C}$ with thread-level parallelisation for computing gradients. Initial field configurations were generated using python scripts. Usually, an initial field configuration was computed on an $N=40$ grid with lattice spacing $h=0.2$. This makes rapid exploration of the solution space possible; the computation 
time to generate relaxed solutions on current Desktop PCs is a few hours. One should make sure all major relaxation is finished and no structural changes are going on before making further refinements to improve energy estimates.

A common method to allow for lattice effects is to correct energies by a factor of $B / B_{\text {num }}$, so that

$$
E=\frac{B}{B_{\text {num }}} E_{\text {num }},
$$

where $E_{\text {num }}$ is the numerical energy, and $B_{\text {num }}$ is the baryon number obtained as the numerically evaluated integral of the baryon density (1.4) using the same discretisation scheme as for the energy. $B$ is the (true) integer baryon number and $E$ is the corrected energy estimate. This method was often used to estimate Skyrmion energies [24], and works surprisingly well in the massless pion case, because the numerical calculations appear to underestimate both the energy and the baryon number by a very similar factor. However, it does not work well for Skyrmions with massive pions. The reason is that the pion mass contribution to the energy is not similarly underestimated by numerics, probably because it does not contain any derivatives. One could try to develop a new extrapolation method, separating derivative and non-derivative energy contributions, but we haven't tried this.

Instead, to get accurate Skyrmion energies, we gradually increased $N$ and gradually decreased $h$, so that the effects of both could be estimated, and an extrapolation to the continuum limit made. This gives both a good energy estimate and an idea of the precision. The Skyrmion energies are presented in Table1, the accuracy is \pm 0.01 . This method works well for massive pions, because the Skyrme field decays quickly (exponentially as $\exp (-m|\mathbf{x}|)$, with $m=1$ here), but would be less useful for massless pion computations, as the Skyrme field decays only algebraically.

\section{References}

[1] T.H.R. Skyrme, A nonlinear field theory. Proc. Roy. Soc. A260 (1961) 127-138.

[2] N. Manton and P. Sutcliffe, Topological Solitons (Chapter 9). Cambridge University Press: Cambridge, 2004.

[3] G.E. Brown and M. Rho, The Multifaceted Skyrmion. World Scientific: Singapore, 2010 .

[4] G.S. Adkins and C.R. Nappi, The Skyrme model with pion masses. Nucl. Phys. B233 (1984) 109-115.

[5] R.A. Battye and P.M. Sutcliffe, Skyrmions with massive pions. Phys. Rev. C73 (2006) 055205. 
[6] R.A. Battye, S. Krusch and P.M. Sutcliffe, Spinning Skyrmions and the Skyrme parameters. Phys. Lett. B626 (2005) 120-126.

[7] R.A. Battye, N.S. Manton, P.M. Sutcliffe and S.W. Wood, Light nuclei of even mass number in the Skyrme model. Phys. Rev. C80 (2009) 034323.

[8] R.A. Battye and P.M. Sutcliffe, Skyrmions, fullerenes and rational maps. Rev. Math. Phys. 14 (2002) 29-85.

[9] R.A. Battye and P.M. Sutcliffe, Skyrmions and the pion mass. Nucl. Phys. B705 (2005) 384-400.

[10] R.A. Battye, N.S. Manton and P.M. Sutcliffe, Skyrmions and the $\alpha$-particle model of nuclei. Proc. Roy. Soc. A463 (2007) 261-279.

[11] C.J. Houghton, N.S. Manton and P.M. Sutcliffe, Rational maps, monopoles and Skyrmions. Nucl. Phys. B510 (1998) 507-537.

[12] N.S. Manton and B.M.A.G. Piette, Understanding Skyrmions using rational maps. Progr. Math. 201 (2001) 469-479.

[13] M. Kugler and S. Shtrikman, A new Skyrmion crystal. Phys. Lett. B208 (1988) 491-494.

[14] M. Kugler and S. Shtrikman, Skyrmion crystals and their symmetries. Phys. Rev. D40 (1989) 3421-3429.

[15] L. Castillejo, P.S.J. Jones, A.D. Jackson, J.J.M. Verbaarschot and A. Jackson, Dense Skyrmion systems. Nucl. Phys. A501 (1989) 801-812.

[16] N.S. Manton, Classical Skyrmions - static solutions and dynamics. Math. Meth. Appl. Sci. 35 (2012) 1188-1204.

[17] N. Furutachi, M. Kimura, A. Dot, Y. Kanada-En'yo and S. Oryu, Cluster structures in oxygen isotopes. Prog. Theor. Phys. 119 (2008) 403-420.

[18] W.K. Baskerville, Making nuclei out of the Skyrme crystal. Nucl. Phys. A596 (1996) 611-630.

[19] D.T.J. Feist, Interactions of $B=4$ Skyrmions. JHEP 2012 : 100.

[20] F. Klein, Lectures on the Icosahedron. Dover Publications: Mineola, N.Y., 2003.

[21] S.W. Wood, Skyrmions and Nuclei. Cambridge University PhD thesis, unpublished, 2009.

[22] J. Shewchuk, An introduction to the conjugate gradient method without the agonizing pain. http://www.cs.cmu.edu/ quake-papers/ painless-conjugate-gradient.pdf, 1994. 
[23] C. Barnes, W.K. Baskerville and N. Turok, Normal mode spectrum of the deuteron in the Skyrme model. Phys. Lett. B44 (1997) 180-186.

[24] R.A. Battye and P.M. Sutcliffe, Symmetric Skyrmions. Phys. Rev. Lett. 79 (1997) 363-366. 